\title{
INTRAMURAL LEIOMYOMAS OF THE BLADDER IN ASYMPTOMATIC MEN
}

\author{
ROBERTO I. LOPES, ROBERTO N. LOPES, MIGUEL SROUGI \\ Women's Beneficent Society, Syrian and Libyan Hospital, São Paulo, SP, Brazil
}

\begin{abstract}
Bladder leiomyomas are rare benign mesenchymal tumors, which account for less than $0.43 \%$ of all bladder tumors with approximately 200 cases described in the literature. These tumors may be classified into 3 different locations: endovesical, intramural and extravesical. Endovesical is the most common form, accounting for $63-86 \%$ of the cases, while intramural occurs in 3-7\% and extravesical in $11-30 \%$.

The intramural form, especially small tumors, may not produce symptoms hardening detection. We report two cases of intramural bladder leiomyomas in asymptomatic men observed incidentally by transabdominal ultrasonography during the follow-up of benign prostatic hyperplasia.

We discuss the diagnosis and management of these lesions.
\end{abstract}

Key words: leiomyoma; bladder; benign neoplasm

Int Braz J Urol. 2003; 29: 245-247

\section{INTRODUCTION}

Bladder leiomyomas are rare benign mesenchymal tumors that account for less than $0.43 \%$ of all bladder tumors (1). Approximately 200 cases have been described in the literature (1).

\section{CASE REPORTS}

Case 1 - 59 year-old man with a 3 -year history of benign prostatic hyperplasia without clinical manifestations. During follow-up, a pelvic ultrasonography demonstrated a well-circumscribed hypoechoic mass at the postero-superior bladder wall measuring 1.74 x $1 \mathrm{~cm}$ (Figure-1). Cystoscopy demonstrated a lesion covered with normal bladder mucosa. A transurethral resection was performed and the pathologic examination revealed a leiomyoma. No recurrence was observed after 10 months.
Case 2 - A 59 year-old asymptomatic man had been accompanied for benign prostatic hyperplasia for 9 years. Transabdominal ultrasonography revealed a $2.8 \times 2.2 \times 1.8 \mathrm{~cm}$ well-circumscribed hypoechoic mass at the antero-superior bladder wall thought to be an urachal cyst, due to its midline location. Computed tomography scan showed bilateral renal cysts and a lesion at the bladder apex (Figure2). Open segmental resection was performed for the latter and pathologic examination revealed leiomyoma. There has been no evidence of recurrence after 10 months.

\section{COMMENTS}

Bladder leiomyomas have been reported to occur equally in both men and women (2). However, more recently a review demonstrated predominance in women (3), which may be attributed to the in- 


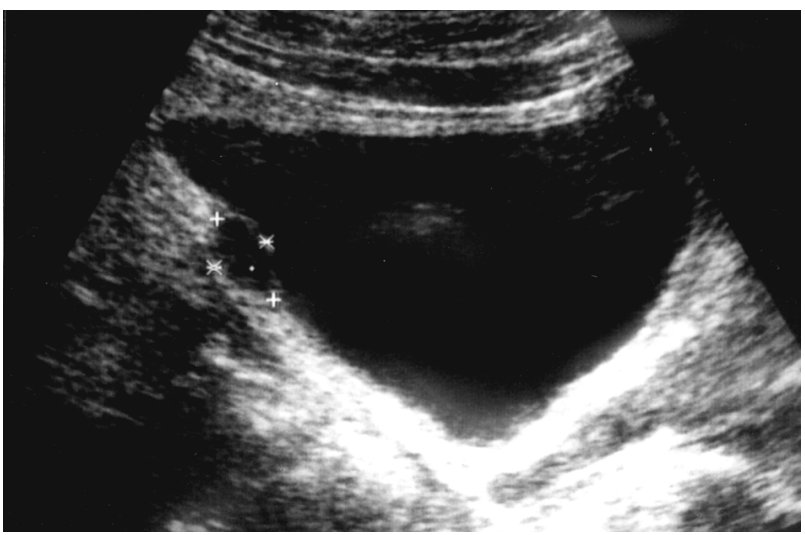

Figure 1 - Ultrasonography (longitudinal scan) demonstrating the bladder tumor (intramural leiomyoma).

creased use of pelvic ultrasonography in female patients (1). In our 2 cases, pelvic ultrasonography performed during follow-up of benign prostatic hyperplasia led to incidental diagnosis of bladder leiomyomas, suggesting that the reported predominance of these tumors in women is questionable.

These tumors may be classified into 3 different locations: endovesical, intramural and extravesical. Endovesical is the most common form, corresponding to $63-86 \%$ of the cases, while intramural occurs in $3-7 \%$, and extravesical in $11-30 \%$ $(2,3)$. Based on cystoscopic findings, an intramural leiomyoma can be distinguished from an endovesical tumor. Endovesical tumors are usually pedunculated or polypoid, while intramural myomas are usually well encapsulated and surrounded by bladder wall muscle.

The endovesical form usually causes irritative or obstructive symptoms and gross hematuria (2) that result in detection (1). Intramural form, especially small tumors, may not produce symptoms.

Radiologically, leiomyomas appear as wellcircumscribed hypoechoic masses at ultrasonography and as in case 2 , these tumors may be misinterpreted as other bladder lesions such as an urachal cyst when observed in a bladder midline position. To rule out other benign lesions and, especially, bladder cancer, the tumor should be biopsed.

Intramural tumors may be managed according to their size and location. Small easily accessible tumors may be treated with transurethral resection, while unfavorable positioning and recognition difficulties may require segmental resection as in case 2 .

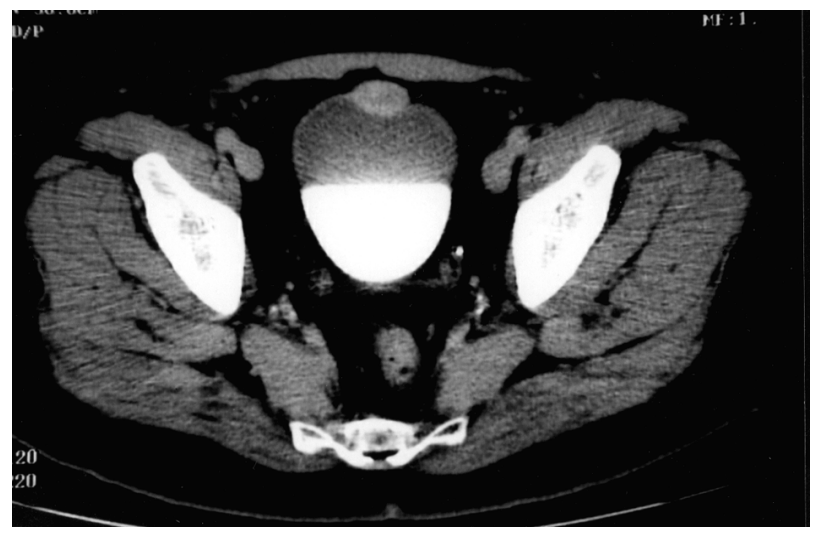

Figure 2 - Computed tomography demonstrating a midline intramural leiomyoma at the bladder dome.

Management of unfavorable lesions comprises open segmental resection or laparoscopic partial cystectomy.

Histopathologically, leiomyoma of the bladder is composed of fascicles of smooth muscle fibers separated by connective tissue. The etiology of these tumors remains unknown. It is proposed that bladder leiomyomas may arise from chromosome abnormalities (1), hormonal influences, bladder musculature infection, perivascular inflammation or dysontogenesis (3).

\section{REFERENCES}

1. Cornella JL, Larson TR, Lee RA, Magrina JF, Kammerer-Doak D: Leiomyoma of the female urethra and bladder: report of twenty-three patients and review of the literature. Am J Obstet Gynecol. 1997; 176:1278-85.

2. Knoll LD, Segura JW, Scheithauer BW: Leiomyoma of the bladder. J Urol. 1986; 136:906-8.

3. Goluboff ET, O'Toole K, Sawczuck IS: Leiomyoma of bladder: report of case and review of literature. Urology 1994; 43:238-42.

Received: January 10, 2003 Accepted after revision: April 14, 2003

Correspondence address:

Dr. Roberto Iglesias Lopes

Rua Baronesa de Itu, 721 / 121

São Paulo, SP, 01231-001, Brasil

Fax: + $55113666-8266$

E-mail: robertoiglesias@ terra.com.br 


\section{EDITORIAL COMMENT}

Leiomyomas of the bladder is distinctly unusual as the author's report. They provide a concise case report of 2 men who were discovered to have this unusual lesion of the bladder.

They provide a wide range of incidences for the 3 locations for a leiomyoma of the bladder. It would seem that given the paucity of this tumor, that it would be difficult to indicate other than the most common location, is what they term endovesicle. I am not even certain what they mean by endovesicle and how they differentiate this from intramural with any precision.
It would seem that an important part of this manuscript, which is overlooked, is whether one can make the diagnosis based upon radiographic configuration and avoid any surgery. The authors do not provide this as an option and simply state that there are several ways of removing these tumors. Since this is a benign neoplasm and if there are no signs or symptoms, one would wonder why it would be necessary to remove the lesion. For instance, if a percutaneous biopsy was performed and the diagnosis was a benign leiomyoma, would it be necessary to proceed with any further surgery, such as removal?

Dr. Mark S. Soloway Professor and Chairman of Urology University of Miami School of Medicine Miami, Florida, USA

\section{REPLY BY THE AUTHORS}

The term endovesical refers to the submucosal growth of leiomyoma, first described by Campbell \& Gislason (1). The endovesical (submucosal) tumors are usually pedunculated or polypoid, while intramural leiomyomas are surrounded by the musculature of the bladder wall (as in these 2 cases reported) and are usually well encapsulated. Distinction between these 2 types is based on cystoscopic findings.
The Authors 